\title{
Das Prinzip Natürlichkeit in Deutsch als Zielsprache: Bemerkungen zur Wörtlichkeit in der zielsprachlichen Wiedergabe von Phraseologismen als Strategie der Übersetzung von Prosatexten
}

\section{The Principle of Naturalness in German as a Target Language: Observations on Literal Translation in the TL-Rendering of Phraseological Units as a Translation Strategy for Prose Texts}

\author{
Pawet Bąk \\ INSTYTUT FILOLOGII GERMAŃSKIEJ, UNIWERSYTET RZESZOWSKI \\ AL. REJTANA 16C, 35-959 RZESZÓW \\ wort.pb@wp.pl
}

\begin{abstract}
In translations of literary works, recipients of the target text know only the results of the translation process; readers are unfamiliar with the original and are unaware of the translation's deviations from the original text, as well as the fate of source-language phraseological units. In a translated text, only word-for-word translations and subsequently unnatural-sounding passages stand out. They seem artificial and non-literary even to readers that do not know the original. It is therefore reasonable to use the criterion of naturalness when discussing and assessing translation solutions. The "invisible" effects of a neutralization strategy for dealing with phraseological units may become apparent here, especially in descriptions of protagonists in the source-language work. The phraseological features that especially stand out when editing a translation are expressions translated word-for-word that have an artificial effect. In this context, interlingual comparison appears to be extremely helpful. This issue is addressed using the example of Henryk Sienkiewicz's novel Bez dogmatu (Without Dogma), a work nearly forgotten today.
\end{abstract}

\section{Einführung in die Problematik}

Dem Modell des Bildfeldes Harald Weinrichs, d.h. der Auffassung von Gemeinsamkeiten bildhafter Wendungen, kann man entnehmen, dass sprachliche Bilder somit auch Phraseologismen mit dem bildhaften Charakter, in verschiedenen Sprachen des Abendlandes nachvollziehbar sind (Weinrich 1976). Nicht alle werden dabei gleich versprachlicht. Die Sprachwissenschaftler weisen auf semantische Unterschiede zwischen formähnlichen Lexemen in zwei Sprachen 
hin, wobei - wie von Lipczuk (2000: 14) und Piirainen (2001: 151-169) angesprochen - manche Phraseolexeme als falsche Freunde des Übersetzers (Tautonyme) gelten können.

Ohne Anspruch auf die Vollständigkeit der Darstellung aller Aspekte der Phraseologie und ohne auf zahlreiche translatologische Einzelfragen einzugehen, wird in vorliegenden Überlegungen versucht, aufzuzeigen, wie die Übersetzer, auf der Suche nach Entsprechungen im idiomatischen Bereich, sich zwischen zwei Polen der Äquivalenz bewegen. Es wird untersucht, ob die Übersetzer die Natürlichkeit der Sprache anstreben oder auf die Ungezwungenheit der Wirkung des Translats (und gegebenenfalls mit welchen Folgen) verzichten. In der Übersetzung ins Deutsche, die im Folgenden zur Sprache kommt, werden einerseits funktionale bzw. dynamische translatorische Lösungen und andererseits Fälle der (zumeist beanstandeten) Wörtlichkeit verzeichnet. Unter der Wörtlichkeit wird - Anette Kopetzki (1996: 330) paraphrasierend - der Vorrang des Einzelwortes (auch des Synsemantikons) vor dem Phraseolexem verstanden. Das breite Spektrum von Übersetzungsmöglichkeiten umfasst mehrere translatologische Verfahren (u.a. als direkte oder kreative Methoden, die Substitution oder Kompensation etc.), ${ }^{1}$ die in der Literatur - neben dem Begriff der Äquivalenz - breit abgehandelt wurden. (S. z.B. Albrecht 1990: 71-81.)

Das Ziel der Überlegungen ist es, die Vorgehensweise bei der Translation und die Auswirkungen von übersetzerischen Entscheidungen auf die ZS-Gestaltung von sprachlichen Charakteristika im Bereich der idiomatischen Einheiten am Beispiel des individuellen Sprachgebrauchs des Protagonisten im Prosawerk Bez dogmatu (Ohne Dogma) von Henryk Sienkiewicz zu beleuchten. Damit soll ein wenn auch bescheidener - Beitrag zur Erforschung von Phraseologismen als Übersetzungsproblem und zur Diskussion über die translatorische Kompetenz (bzw. deren Teilkompetenzen) geleistet werden. ${ }^{2}$

Während konkrete Wendungen, z.B. phraseologische Einheiten, ohne weiteres sprachliche Erscheinungen sind, betreffen die hier angesprochenen Aspekte der Entscheidungen des Übersetzers, das translatorische Handeln, also die übersetzerische Pragmatik.

Des Öfteren wird das ebenso allgemeine wie auch unpräzise Merkmal natürlich für die Bewertung von Translaten herangezogen. Übersetzungen literarischer Texte haben den Status eigenständiger Werke - sie werden ohne die Kenntnis von Übersetzungsvorlagen rezipiert. Das Translat hat somit die Aufgabe, beim Leser den Eindruck zu wecken, dass er es mit dem Original zu tun hat. Nicht zufällig wird das Prinzip Natürlichkeit im Folgenden zur Sprache gebracht. Wir haben es hier nämlich mit einem Roman als Übersetzungsvorlage zu tun, in dem Ungewöhnlichkeiten der Ausdrucksweise den Mittelpunkt von sprachlichen Charakteristika des Protagonisten bilden. ${ }^{3}$ Schon im Falle des Originals erscheint

1 Bei anderen Autoren als Prozeduren oder Techniken betrachtet. Unter den Übersetzungsergebnissen im Bereich der Phraseologie (u.a. Idiome, Zwillingsformeln) kann man verschiedene Arten der übersetzerischen Technik feststellen.

${ }^{2}$ Zum Begriff der translatorischen Kompetenz siehe vor allem bei Grucza (1993), ferner auch bei Risku (1998).

3 Im Folgenden wird nicht an alle Erscheinungsformen des Natürlichen in der Linguistik, z.B. als Prinzipien der Natürlichkeitstheorie, angeknüpft. 
die Frage nach der Natürlichkeit der Wirkung als ein Anlass zur Diskussion über komplexe Phänomene.

Es wird hier versucht, unter den bisher präzise nicht definierten Kriterien der Akzeptanz von Übersetzungslösungen (im Bereich der Idiomatik) auch den (Bei)Wert Natürlichkeit zu beachten, um ihn auf die Nützlichkeit als Kriterium der Bewertung von Translaten hin zu überprüfen. ${ }^{4}$

Die Bildhaftigkeit ist stark mit der Motiviertheit (oder Motivierbarkeit) und der Bekanntheit (Lexikalisiertheit) der Phraseolexeme verbunden. Auch die Aura der Zeit (die sprachlich-literarische Konvention) soll dabei mitbeachtet werden. Damit hängt auch die Expressivität der Wendungen zusammen, die allerdings nicht ein konstantes Merkmal ist. Sie wird hier vielmehr als Gegenstand der Parole verstanden, d.h. eine Eigenschaft, die im jeweiligen Kontext aktualisiert werden kann. 5

Die Problematik hat mit mehreren Merkmalen solcher Wendungen, u.a. mit der Bildhaftigkeit ${ }^{6} \mathrm{zu}$ tun. Sie berührt auch die Frage, ob Wörter richtig zu phraseologischen Wendungen verknüpft und im vorgegebenen Kontext angemessen verwendet werden.

Diese Fragen samt der Beachtung der Kompatibilität von Bestandteilen ${ }^{7}$ und ihre Wahrung in der Translation werden im Folgenden im Zusammenhang mit dem Begriff Natürlichkeit betrachtet.

\section{Zum Roman Bez dogmatu: (Un)Natürlichkeit der Sprache}

Bez dogmatu (Ohne Dogma), ein Gegenwartsroman von Henryk Sienkiewicz (1891), ${ }^{8}$ hebt sich von den anderen Werken des Nobelpreisträgers ab, die dem Autor zu seinem großen Ruhm verholfen haben. Für seine bedeutendsten Romane wählte Sienkiewicz meistens geschichtliche Sujets. Mit Bez dogmatu wollte er gewissermaßen ein Urteil über die Menschen seiner Zeit fällen und lieferte - wie von Krzyżanowski (1966) behauptet - den Beweis für das Talent, psychologischsoziale Romane zu verfassen. 9

Bez dogmatu hat die Form eines Tagebuches, in dem die Überlegungen des Hauptprotagonisten breiten Raum einnehmen. Im Roman findet man mehrere phraseologische Wendungen, Zwillingsformeln, Redensarten, erstarrte Phraseologismen - auch Einheiten, die der höchste Grad der semantischen Verschmelzung kennzeichnet. Der Gebrauch solcher Ausdrücke dient der

\footnotetext{
4 Es wird hierbei jedoch nicht darauf abgezielt, die Ergebnisse der übersetzerischen Tätigkeit zu bewerten. Der übersetzungskritische Blick und Angabe von Alternativvorschlägen sind hier allerdings (wie auch sonst im Falle von Übersetzungsanalysen oft) nicht zu meiden.

5 Ähnliches fokussiert Labno-Falęcka, die von der Expressivität und Motiviertheit (Motivierbarkeit) als potentiellen Charakteristika des Phraseologismus in einer konkreten Situation (Epoche etc.) spricht. (S. Labno-Fałęcka 1995: 221.)

${ }^{6}$ Labno-Fałęcka spricht hierbei von Anschaulichkeit und Belebungsprozessen (s. ebenda).

7 Ähnliches gilt für die Wahrung der Kollokation bei nichtidiomatischen Wortverbindungen, z.B. in der Verknüpfung von Autosemantika zu freien oder losen Verbindungen.

${ }^{8}$ Deutsch erstmals 1910. Die letzte Übersetzung von Christa und Johannes Jankowiak aus dem Jahre 1989 wird im Folgenden angesprochen.

9 Sienkiewicz selbst stellte fest, dass er einen psychologischen Roman geschrieben habe. (S. Krzyżanowski 1966: 151.)
} 
Charakterisierung der Gestalt und prägt - neben anderen Ausdrucksmitteln - die Sprache des Helden.

Die Betrachtungsperspektive ist dabei durchaus kompliziert. Die Problematik betrifft die Natürlichkeit der Wirkung, während die Ausdrucksweise des Protagonisten - der Gegenstand des translatorischen Transfers - bereits in der Übersetzungsvorlage nicht immer viel Natürlichkeit ausstrahlt: Manche Wendungen wirken bereits im Original unnatürlich. Es wird in diesem Zusammenhang die Frage gestellt, ob dies den Übersetzer dazu berechtigt, auf das Prinzip Natürlichkeit schlechthin zu verzichten.

Leon Płoszowski, die Hauptfigur des Romans, kam in Italien zur Welt - in dem Land, das sein Vater zur Heimat wählte. Er ist ein entwurzelter Weltbürger, aus- und eingebildeter reicher Mann, Ästhet und Skeptiker, dem während eines Aufenthalts in Polen eine große Liebe zuteil wird. Der Weltmann, der viele Abenteuer hinter sich hatte (abgesehen von kurzen Besuchen in Warschau, verbrachte er sein Leben zwischen Rom und Paris), wird durch die Anmut der Polin Anielka bezaubert, als er ihr nach Jahren seiner Abwesenheit hierzulande wieder begegnet. Als weltentfremdetes, verwöhntes und willensschwaches Individuum, das auf sich selbst bezogen den Ausdruck l'improductivite slave (S18) gelten läßt, wird Płoszowski zu einem pessimistisch gesinnten Dilletanten des polnischen Fin de Siècle stilisiert. ${ }^{\circ}$ Seine Geschichte (in der Ich-Form erzählt) endet tragisch: Die Geliebte, die einem anderen gehört, stirbt. Er wählt den Freitod. Eine auf den ersten Blick banale Handlung wird um das Motiv der unglücklichen Liebe mit dem Selbstmord als Ausgang aufgebaut. Die Sprache, deren sich Leon Płoszowski in seinem Tagebuch bedient, unterscheidet sich vom geläufigen Sienkiewicz-Stil. Bestimmte charakteristische Züge in der Ausdrucksweise haben es dem Autor ermöglicht, einen anderen Helden als den in seiner Trilogie zu formen, zu dem der Autor im Text keine Distanz herstellt.

Płoszowski gebraucht manche Wörter und Wendungen auf eine Art und Weise, dass diese dem Leser auffallen müssen. Es sind (Einzel- und Phraseo)Lexeme, die den individuellen Sprachgebrauch kennzeichnen. Die ausgefallene Sprechweise des Protagonisten äußert sich u.a. im Anhäufen von Okkasionalismen, Fremdwörtern, Phraseologismen, Bibelwörtern, falsch gebrauchten Fachtermini - all dies in einer bunten Mischung. Viele Ausdrücke, deren sich dieser gebildete Mann bedient, wirken unangemessen, sind in manchen Zusammenhängen überflüssig, fehl am Platze - kurz gesagt: Sie wirken unnatürlich. Besonders fallen bei der Lektüre Phraseologismen auf, die kreativ verwendet werden, z.B. wenn deren Glieder durch Abwandlungen neue Kollokationen eingehen ${ }^{11}$. Es handelt sich dabei nicht um Modifizierungen im Sinne von Sprachspielen, sondern um Wendungen, die gespreizt, gekünstelt wirken, weil sie „fremde“ Bestandteile enthalten. Gehobenes wird von Płoszowski mit Saloppem vermischt, die Termini werden miteinander verwechselt oder zueinander synonym verwendet. Beispielsweise gebraucht er zur Bezeichnung der Aquädukte den Ausdruck wodociagi (S11), der keineswegs an Baudenkmäler der

\footnotetext{
10 Darüber sind sich nicht alle Literaturforscher einig. Einen neuen Blick auf den Protagonisten warf kürzlich Rozpłochowska. In ihrer Studie legt sie nahe, Płoszowski als keinen eindeutig dekadenten Charakter zu betrachten. (S. Rozpłochowska 2006: 117-142.)

${ }_{11}$ Mehr zu okkasionellen Bildungen und Neologismen als Idiolektes von Płoszowki wurde in Bąk (1997: 47) angesprochen.
} 


\section{Pawet Bąk: Das Prinzip Natürlichkeit in Deutsch als Zielsprache}

altertümlichen Architektur denken lässt. Über Aserbaidschan spricht er, indem er sich des irreführenden geographischen Namens Daleki Wschód (S190) bedient, das ins Deutsche von Jankowiak nicht als der Ferne Osten, sondern mit der korrigierenden Generalisierung der Osten (J. 237) übersetzt wurde. Die Übersetzer Christa und Johannes Jankowiak erlagen der Versuchung, solche Quasifehler zu korrigieren. Wodociagi wurde von ihnen als Aquädukte (J14) wiedergegeben.

Die ausgefallene Sprechweise Leon Płoszowskis ist mit dem häufigen Auftreten okkasioneller Bildungen verbunden, auch solcher Ausdrücke, die im allgemeinen Wortschatz jener Zeit nicht auftraten. ${ }^{12}$

Neben auffälligen Merkmalen spielen Phraseologismen eine wichtige Rolle bei der literarischen Gestaltung des Protagonisten im Text. Er wird durch ihren Reichtum sprachlich charakterisiert. Der Autor, Henryk Sienkiewicz, lässt die Ausdrucksweise, voller Fremdwörter, Sentenzen etc., der sozialen Position des Helden entsprechen. Beachtenswert ist dabei die Tatsache, dass Sienkiewicz der Hauptfigur nicht nur in Dialogen, sondern auch im Monolog, der das gesamte Tagebuch durchzieht, extravagante Ausdrücke in den Mund legt. Der letzte Umstand mag möglicherweise davon zeugen, dass sich Sienkiewicz mit dem literarisch kreierten Płoszowski identifiziert.

Die Ausdrucksweise in der Übersetzungsvorlage, d.h. ihre unnatürliche Wirkung, mag dem Übersetzer mehr Freiheit (und mehr kreativen Spielraum) zur Verfügung stellen, als sonst bei der Wiedergabe von festen Wendungen zu erwarten ist. Neben den Aspekten des individuellen Sprachgebrauchs, Problemen mit der Abgrenzung des Phraseologischen vom Innovativen ${ }^{13}$ muss bei der Betrachtung der Sprache auch die Aura der Zeit beachtet werden (in Anlehnung an Wörterbücher, z.B. M. Arcta Stownik frazeologiczny: poradnik językowy, 1934). All dies verlangt vom Übersetzer viel Verantwortungsbewusstsein. Er muss an phraseologische Wendungen als „fremdes Wortgut“ (d.h. Produkt eines anderen Autors) adäquat herangehen, also zunächst die lexikalisierten Wendungen von innovativen (neuen, überraschenden) oder falschen abgrenzen und mit analogen Lexemen übersetzen.

Das Prinzip Natürlichkeit erscheint hier im doppelten Sinne. Es bezeichnet einerseits im Original unnatürlich wirkende Äußerungen. Sie sollten vom Übersetzer als solche erkannt werden. Andererseits drängt sich beim Vergleich des Ausgangstextes und der Übersetzung die Frage auf, ob das Translat „natürlich“ zu wirken hat, wenn der ausgangssprachliche Text Unnatürlichkeit ausstrahlt, und ob illusionistische Methoden (etwa im Sinne Jiří Levýs, 1969) dann die Übersetzung nicht allzu stark von der Übersetzungsvorlage entfernen. Als eine Alternative könnte der Fall gelten, wo beim Übersetzen kontroverse sprachliche Charakteristika (z.B. Stilbrüche) vom Ausgangstext übernommen werden, auch wenn der zielsprachliche Leser diese nicht immer als begründet und äquivalent erkennt.

In diesem Zusammenhang kommt noch die Frage auf, ob das genaue sprachliche Bild des Protagonisten zu den Invarianten gehört oder ob - dem

\footnotetext{
12 Dies wurde anhand des phraseologischen Wörterbuchs M. Arcta Stownik frazeologiczny: poradnik językowy (1934) überprüft. Die Archaismen und Historismen werden im Folgenden nicht angesprochen. Auch die diachrone Betrachtung der Phraseologie kann hierbei nicht ausführlicher erfolgen.

${ }^{13}$ Mehr dazu bei Pajdzińska (2006: 222-231).
} 
Prinzip illusionistischer Methoden zufolge - eventuelle Neutralisierungen (Verluste) beim Lesen der Übersetzung den Eindruck erwecken können, man lese das Original.

Im Rahmen einer anderen Analyse (Bąk 1997) wurde belegt, dass die Übersetzer Ungewöhnlichkeiten der idiolektalen Formulierungen im ZT nicht beibehalten und sich in der Mehrheit von Fällen für Neutralisierungen entschieden. Im Folgenden wird der Frage nachgegangen, ob die translatorische Strategie auch im Bereich der Phraseologie, so wie in anderen Fällen, Neutralisierungen zulässt und ob die Übersetzer solche Neutralisierungen konsequent einsetzen (bzw. zulassen) oder in dieser Hinsicht selektiv (bzw. arbiträr) vorgehen. Dies wird anhand der Beispiele untersucht, die aus der Übersetzung von Christa und Johannes Jankowiak (1989) stammen. Diese Übertragung stellt heute gegenüber den Übersetzungen von Kotulski und Winikoff (beide um 1910 veröffentlicht) und der Kroczek-Übersetzung aus dem Jahre 1928 die zugänglichste deutsche Fassung von Bez dogmatu dar.

\section{Die Übersetzung von Phraseologismen in Bez Dogmatu}

Im Vorliegenden werden einige Resultate der Translation an Beispielen veranschaulicht. Im Rahmen eines größer angelegten Übersetzungsvergleichs mit deskriptivem Charakter kann eine genauere Typologie der Übersetzungsergebnisse im Bereich der Phraseologie als Charakteristikum des individuellen Sprachgebrauchs vorgenommen werden.

Dem Prinzip der Bildfelder (im Sinne Harald Weinrichs) entsprechend, lassen sich im Zieltext viele direkt übersetzte Phraseolexeme finden, ${ }^{14}$ die im ZT natürlich wirken. Es sind lexikalisierte bildhafte metaphorische Wendungen, die - Weinrich zufolge - nicht nur innerhalb einer Sprachgemeinschaft leicht nachvollziehbar, sondern auch in fremde Sprachen übertragbar sind:

W rozprawy wdawać z tobą się nie mogę, bo mnie w kozi róg zapędzisz. (S263)

In Debatten mit dir kann ich mich nicht einlassen, weil du mich ständig ins

Bockshorn jagst. (J324)

Można też było głowę stracić. (S31)

Man konnte [...] schon den Kopf verlieren. (J40)

obrót rzeczy (S60)

Wendung der Dinge (J79)

trzymać nerwy na wodzy (S87)

Nerven im Zaume halten (J111)

Ten gatunek Polek woli złamać serce niż słowo. (S122)

Dieser Typ der Polinnen würde eher das Herz des Anderen brechen als ihr Wort. (J153)

${ }^{14}$ Im Folgenden werden u.a. Redewendungen, Redensarten etc. zusammen aufgefasst. 
rozdrapywać własne rany (S144)

die eigenen Wunden aufreiße[n] (J181)

Darunter sind in der Übertragung von Ch. und J. Jankowiak solche Wendungen als Entsprechungen zu finden, die auf der gleichen begrifflichen (konzeptuellen oder kulturbedingten) Basis bzw. - gemäß dem Weinrichschen Modell - innerhalb desselben Bildfeldes entstanden sind, obwohl manche von ihnen in Deutsch als $Z S^{15}$ gewissen Modifizierungen unterliegen. Sie betreffen hauptsächlich die Form, weisen aber auch zum Beispiel eine andere Distribution oder abweichende Kollokationen auf. Geringfügige (formale) Abweichungen vom polnischen Wortlaut der Phraseolexeme sind hier nicht das Ergebnis von Eingriffen des Übersetzers in die sprachliche Gestalt der Translate, sondern vielmehr eine Bestätigung der These, dass sich die Phraseologie des Abendlandes im Bereich derselben Bildfelder entwickelt(e), auch wenn sie wegen formaler Charakteristika von Sprachen und durch zusätzliche Abwandlungen, Umdeutungen etc. jeweils voneinander divergierende sprachliche Gestalten annehmen. So übersetzte zielsprachliche Wendungen, deren Übersetzung die Kenntnis der gleichen Begriffe (Konzepte) erfordert, können als direkte Äquivalente betrachtet werden. ${ }^{16}$

Manche Entsprechungen weisen jedoch Abweichungen von den Wendungen im Original auf. Trotz der Unterschiede kann man dabei oft noch davon sprechen, dass die Wendungen im AT und ZT innerhalb desselben begrifflichen Rahmens bleiben: Sie unterscheiden sich nur durch eine andere formale Repräsentation derselben semantischen Funktion, z.B. der gleichen Handlungsträger- oder Instrumentrelationen oder einer anderen Beziehung innerhalb desselben begrifflichen Bereiches:

$$
\text { coś upatrzonego [mieć] (S23) }
$$

etwas im Auge [haben] (J30)

Serce się kraje. (S84)

Mir blutet das Herz. (J107)

bez ogródek mówić (S9)

ohne ein Blatt vor den Mund zu nehmen [sprechen] (J11)

Nikomu nie przyszło do głowy [...] (S12)

Niemandem kam es in den Sinn (J15)

Z tego błędnego koła nie wyjdziesz, więc w nie nie wchodź. (S20)

Aus diesem Teufelskreis kommst du nicht heraus, also betritt ihn nicht erst

15 Mit dieser Formulierung wird an den Begriff DaF erinnert: Die fremdsprachdidaktischen Aspekte der Betrachtung von Phraseologismen werden künftig von mir in einem anderen Kontext angesprochen.

${ }^{16}$ Die Beispiele sollten an die von Elisabeth Piirainen im Projekt EUROPHRAS Weit verbreitete Idiome in Europa und darüber hinaus gesammelten Beispiele für phraseologische Volläquivalente in mehreren Sprachen erinnern. Sie stellen auch den Gegenstand kontrastiver Analyse als Interphraseologismen dar, s. Schatte (2006: 143-156). 
(J26)

Nagle zrobiło mi się jasno w głowie. (S27)

Plötzlich dämmerte es mir. (J35)

z szlachetniejszego kruszcu ulany (S248)

aus edlerem Erz gegossen (J308)

za siebie samego nie dałbym obecnie trzech złamanych groszy. (S336)

Für mich als Person gäbe ich gegenwärtig keinen Heller. (J407)

Nie pozwalający sobie nikomu w kaszę dmuchać [...]. (S36)

[Er] läßt sich von niemandem in die Grütze spucken. ${ }^{17}$ (J47)

Die Grenze der letztgenanten Translate zu den funktionalen Äquivalenten ist unscharf. Die folgenden Übersetzungslösungen sind Ergebnisse einer direkten ZSWiedergabe, wobei sie keinerlei Anzeichen einer wörtlichen Wiedergabe aufweisen. ${ }^{18}$ Sie wirken alles in allem natürlich:

Taki, któremu wszystko idzie jak po maśle. (S265)

Einer, bei dem alles glatt geht. (J328)

blada jak papier (S226)

kreidebleich (J280)

Więc pal ich licho! (S42)

Also hol sie der Teufel! (J55)

Pal ją licho! (S95)

Sie soll sich zum Teufel scheren! (J120)

Wodziłem więc rej w całej szkole. (S12)

[Ich] spielte [...] in der Schule die erste Geige. (J15)

wyprowadzony w pole (S15)

hinters Licht geführt (J2O)

Die Bildhaftigkeit der Sprache des Originals im phraseologischen Bereich geht in der deutschen Sprache an einigen Stellen verloren: Phraseologische Einheiten (verschiedener Art) werden als nichtphraseologische Wortverbindungen wiedergegeben. Dieser Tatsache sind sich die Leser jedoch nicht bewusst, denn die folgenden Translate zeichnet eine weitgehende Natürlichkeit aus, die die illusionistischen Methoden des Übersetzens charakterisiert:

${ }^{17}$ In Redewendungen und sprichwörtliche Redensarten: sich nicht auf den Kopf spucken lassen / sich nicht in die Suppe spucken lassen (1992: 678). Zu Problemen mit dem Register im Umgang mit den Phraseologismen s. Labno-Falecka (1995: 198).

${ }^{18}$ Zur Wörtlichkeit s. Kopetzki (1996: 330). 
rozmow[a], którą jednak na razie wziąłem na opak (S69)

Das Gespräch, das ich jedoch zunächst völlig falsch aufnahm (J88)

$$
\begin{aligned}
& \text { tchórzem podszyty ( } \left.\mathrm{S}_{105}\right) \\
& \text { ein ausgesprochener Feigling ( } \mathrm{J} 130)
\end{aligned}
$$

Widocznie moje słowa dotknęły ją do żywego. (S296)

Sichtlich hatten meine Worte sie aufs empfindlichste getroffen. (J364)

Diese Neutralisierungen sind im Translat nicht spürbar. Sie werden als Verluste im Übersetzungsprozess erst im Vergleich des Originals mit dem Translat aufgedeckt. Der Rezipient kann eventuelle Paraphrasen, Substitutionen etc. nicht als solche wahrnehmen. Die Ersetzung idiomatischer Wendungen durch Nicht-Idiome verursacht hier keine Stilbrüche. Der Text strahlt keine Unnatürlichkeit aus. Der Leser könnte sich der Neutralisierungen erst dann bewusst werden, wenn er das Original vor Augen hat.

Vorerwähnte Nichtübereinstimmungen zwischen dem Original und Translat vermag der Rezipient nicht wahrzunehmen. Dies ist allerdings im Falle der nächsten Beispiele zu beobachten, wo in der Übersetzung für kulturspezifische AS-Phraseolexeme wörtliche Entsprechungen benutzt wurden, die auf den ZT-Leser gewissermaßen unnatürlich, verfremdend oder - zumindest - neu wirken.

Beispielsweise kann die Übersetzung rozpędzić na cztery wiatry (S161) mit in alle vier Winde jagen (J203) (wörtlich übertragene Kollokation in alle vier Winde + jagen) als noch nicht unnatürlich angesehen werden. Nur die Kollokation des Verbs mit der lexikalisierten Wortgruppe erscheint hier als neu, der Kern der Wendung (in alle vier Winde) stellt denn aber eine äquivalente Lösung dar. ${ }^{19}$ Die Frage aber danach, was den Übersetzer dazu berechtigt, auf das Prinzip Natürlichkeit zu verzichten, drängt sich bei den folgenden Beispielen auf, die Ergebnisse einer wortwörtlichen Übersetzung sind. Unter vielen findet man folgende, die in der Zielsprache durch die Unnatürlichkeit auffallen:

W swoim czasie byłaby mnie w łyżce wody utopiła za Anielkę. (S178) Seinerzeit hätte sie mich wegen Anielka am liebsten in einem Löffel Wasser ertränkt.20 (J223)

Trafiła kosa na kamień (S204)

die Sense traf auf einen Stein. (J254) ${ }^{21}$

Wiem [...] dlatego, że oddałbym za ciebie ostatnią kroplę krwi. (S194) Ich weiß $[\ldots]$, weil ich für dich den letzten Blutstropfen hingäbe. ${ }^{22}$ (J241)

\footnotetext{
${ }^{19}$ In Duden Redewendungen ist die Wortgruppe in Verbindung mit zerstreuen verzeichnet. (Duden Redewendungen 2002: 872)

${ }^{20} \mathrm{Im}$ Deutschen (besonders jedoch im Jiddischen) ist die Phrase slawischer Herkunft anzutreffen: Er möchte ihn in einem Löffel Wasser ersäufen/ersticken/erwürgen als Bezeichnung der Feindseligkeit und Rache.

${ }^{21}$ Als Alternativvorschlag kann hierfür das Sprichwort Der Krug geht so lange zum Wasser, bis er bricht gelten.

${ }^{22}$ Als die nichtwörtliche Übersetzung empfiehlt sich hier der folgende Wortlaut der Phrase: Ich weiß [...], weil ich für dich mein Herzensblut hingäbe. $\mathrm{Zu}$ sein Herzensblut hingeben $\mathrm{s}$. Redewendungen und sprichwörtliche Redensarten (1992: 339).
} 
Jesteś mu ciężarem i kulą u nogi. (S338)

Du bist ihm eine Last und eine Kugel am Bein. (J410)

Der Alternativvorschlag, der im Gegensatz zum letzten Translat keine Interlinearversion ist, lautet in Anlehnung an Duden - Deutsches Universalwörterbuch: Du bist ihm ein Klotz am Bein. ${ }^{23}$

Die letzt erwähnten (deutschsprachigen) Passagen machen den Eindruck, dass sie sprachliche Neuerungen enthalten. Sie fallen ins Auge. Man kann sie als nicht äquivalent schlechthin, d.h. als überstilisiert empfinden. So auffällige Beispiele wie Die Sense traf auf einen Stein und Kugel am Bein sein lenken die Aufmerksamkeit auf sich und prägen weitgehend die Qualität des Translats. Man kann sie als entweder bildhafter als die lexikalisierten AS-Phrasen oder geradezu als falsch ansehen.

Die Ursachen für solche Übersetzungslösungen können am eventuellen Mangel an sprachlicher Kompetenz der Übersetzer liegen. Man kann sie jedoch nicht nur mit den Kenntnissen der AS und ZS verbinden. Neben der translatorischen Kompetenz spielen bei den Entscheidungen auch Aspekte pragmatischen Charakters eine wichtige Rolle, was in diesen Fällen sichtbar wird. Diese Wiedergabe kann mit der Überzeugung der Übersetzer zusammenhängen, dass sie es mit der Unnatürlichkeit der Ausdrucksweise des Protagonisten in der Übersetzungsvorlage zu tun haben, die primäres Merkmal auch dieser Textstellen ist. Diese Überzeugung bestärkt die Übersetzer darin, das Prinzip der Natürlichkeit aufzugeben.

Der Übersetzer ist bemüht, die Bildhaftigkeit der Äußerungen des Helden beizubehalten, denn er betrachtet diese als die Invariante. Vorerwähnte Neutralisierungen (in verschiedenen Bereichen) könnten in diesem Sinne durch eine adäquate ZS-Gestaltung anderer Lexeme ausgeglichen werden. Die Übersetzungsstrategie verrät in ihren Zügen, dass die Übersetzer so sehr von der Notwendigkeit, wichtige stilistische Komponenten aufrechtzuerhalten, überzeugt waren, dass sie dem Hang zur Einführung neuer Merkmale erliegen. Im Zieltext kann man nun dadurch auch Charakteristika der Sprache finden, für die keine Entsprechungen im Original vorliegen (die letzten Zitate sind Beispiele für Überstilisierungen). Der Übersetzer schneidet dadurch jedoch nicht gerade vorbildlich ab, zumal in seiner Arbeit Inkonsequenzen, Selektivität und Arbitrarität festzustellen sind. Einerseits findet man Neutralisierungen in vielerlei Hinsicht, andererseits die wortwörtliche Wiedergabe im idiomatischen Bereich. Versucht man letztere Übersetzungen als Kompensationsmechanismen zu betrachten, so muss man feststellen, dass die beinahe interlineare Wiedergabe die Verluste in der Übersetzung anderer Bereiche der Sprache nur zum Teil kompensiert und als Strategie keine gelungene Lösung ist.

\section{Schlussbemerkungen}

Bei der Übersetzung eines literarischen Werkes sind dem ZT-Rezipienten die Ergebnisse der Translation bekannt. Er kennt jedoch nicht das Original und

23 Dazu Jemandem ein Klotz am Bein sein bzw. jemandem zur Last fallen (Duden - Deutsches Universalwörterbuch, 1992: 435). 
etwaige Abweichungen der Übersetzung von der Vorlage sowie das Schicksal ausgangssprachlicher Phraseologismen. In diesem Zusammenhang erscheint die Überprüfung des Kriteriums Natürlichkeit bei der Besprechung und Bewertung der translatorischen Leistung als gerechtfertigt.

„Unsichtbare“ Auswirkungen von Neutralisierungen, die das Kriterium Natürlichkeit rechtfertigen könnte, werden - unter Umständen - erst bei der Beschreibung von Protagonisten des AS-Werkes spürbar. Sie fallen im Bereich der Phraseologie ins Gewicht, besonders wenn sie wörtlich übersetzt als nachgeahmte, gespreizte oder geblümte, d.h. künstlich wirkende Redensarten in die Zielsprache kommen. Wortwörtlich übersetzte und dadurch sprachlich unnatürliche Passagen sind im Translat auffällig, denn sie erscheinen dem ZT-Leser auch ohne Kenntnis des Originals als nichtliterarisch. In dem vom Leser rezipierten fertigen Textprodukt können allerdings manche Stilbrüche sowohl als die authentische Ausdrucksweise als auch als translatorische Überstilisierungen und Fehler schlechthin wahrgenommen werden. Was sie tatsächlich sind, wird erst durch den Übersetzungsvergleich aufgedeckt. In diesem Zusammenhang erweist sich der interlinguale Vergleich als aufschlussreich.

Das Natürliche im Originaltext kann nicht immer bei der Übersetzung als absolute Invariante betrachtet werden, besonders wenn die Sprache im AT unnatürlich bzw. exaltiert ist. Das Unnatürliche der Sprache wiederum verlangt vom Übersetzer, vorsichtig mit Auffälligkeiten im Text umzugehen, um eventuelle Überstilisierungen im Translat zu vermeiden. Das Prinzip Natürlichkeit kann auch als Merkmal des Zieltextes in die Irre führen. Mit dem Mantel der so verstandenen Authentizität der Ausdrucksweise können dann Mechanismen verdeckt werden, die die Übersetzung von der Vorlage entfernen.

\section{Abkürzungen}

AS - Ausgangssprache

AT - Ausgangstext

J - Sienkiewicz, dt. von Jankowiak (1989)

S - Sienkiewicz (1978)

ZS - Zielsprache

ZT - Zieltext

\section{Bibliographie}

\section{Literatur mit Siglen}

Sienkiewicz, H. 1978. Bez Dogmatu. Warszawa: Państwowy Instytut Wydawniczy. (=S)

Sienkiewicz, H. 1989. Ohne Dogma. [Dt. v. Ch. u. J. Jankowiak]. Berlin: Rutten \& Loening. (=J)

\section{Sekundärliteratur}

Albrecht, J. 1990. Invarianz, Äquivalenz, Adäquatheit. In: R. Arntz, G. Thome (eds.) Übersetzungswissenschaft. Ergebnisse und Perspektiven. Festschrift für Wolfram Wilss zum 65. Geburtstag. Tübingen: Gunter Narr, 71-81.

Arct, M. 1934. M. Arcta Stownik frazeologiczny: poradnik językowy. Warszawa: Wydawnictwo M. Arcta.

Bąk, P. 1997. Zur Spezifik der literarischen Übersetzung am Beispiel des Romans „Ohne Dogma" von Henryk Sienkiewicz. [Unveröffentlichte Magisterarbeit]. Rzeszów: Wyższa Szkoła Pedagogiczna.

Skorupka, S. 2002. Stownik frazeologiczny języka polskiego. Warszawa: Wiedza Powszechna. 
Duden. 2001. Duden - Deutsches Universalwörterbuch. Mannheim, Leipzig, Wien, Zürich: Bibliographisches Institut.

Duden Bd. 11. 1992. Redewendungen und sprichwörtliche Redensarten: Wörterbuch der deutschen Idiomatik. Mannheim, Leipzig, Wien, Zürich: Bibliographisches Institut \& F.A. Brockhaus AG.

Duden Bd. 11. 2002. Redewendungen. Mannheim, Leipzig, Wien, Zürich: Bibliographisches Institut \& F.A. Brockhaus AG.

Grucza, F. 1993. Interkulturelle Translationskompetenz: Ihre Struktur und Natur. In: A. P. Frank, K. Maaß, F. Paul, H. Turk (eds.) Übersetzen, Verstehen, Brücken bauen. Geisteswissenschaftliches und literarisches Übersetzen im internationalen Kulturaustausch. Band 8, Teil 2. Berlin: Erich Schmidt Verlag. 158-171.

Koller, W. 1997. Einführung in die Übersetzungswissenschaft. Wiesbaden: Quelle \& Meyer.

Krysztofiak, M. 1999. Przekład literacki a translatologia. Poznań: Wydawnictwo Naukowe Uniwersytetu im. A. Mickiewicza w Poznaniu.

Krzyżanowski, J. 1966. Henryka Sienkiewicza żywot i sprawy. Warszawa: Państwowy Instytut Wydawniczy.

Labno-Fałęcka, E. 1995. Phraseologie und Übersetzen: Eine Untersuchung der Übersetzbarkeit kreativ-innovativ gebrauchter „wiederholter Rede“ anhand von Beispielen aus der polnischen und deutschen Gegenwartsliteratur. Frankfurt a.M.: Peter Lang.

Kopetzki, A. 1996. Beim Wort nehmen: sprachtheoretische und ästhetische Probleme der literarischen Übersetzung. Stuttgart: $\mathrm{M}$ und $\mathrm{P}$, Verlag für Wissenschaft und Forschung.

Levý, J. 1969. Die literarische Übersetzung. Theorie einer Kunstgattung. [Dt. von W. Schamschula]. Frankfurt a.M., Bonn: Athenäum Verlag.

Lipczuk, R. 2000. „Fałszywi przyjaciele tłumacza” w słownikach niemiecko-polskich. In: A. Kątny (ed.) Problemy frazeologii $i$ leksykografii [...]. Olecko: Wszechnica Mazurska, 13-21.

Pajdzińska, A. 2006. Studia frazeologiczne. Łask: Oficyna Wydawnicza Leksem.

Piirainen, E. 2001. „Falsche Freunde“ in der Phraseologie: Versuch einer Modellierung. Lexikon und Text. In: H. Nikula, R. Drescher (eds.) Vaasan Yliopiston Julkaisuja. Proceedings of the University of Vaasa 76/4. Vaasa: University of Vaasa, p. 151-169.

Risku, H. 1998. Translatorische Kompetenz - Kognitive Grundlagen des Übersetzens als Expertentätigkeit. Tübingen: Stauffenberg Verlag.

Rozpłochowska, A. 2006. Dekadentyzm utracony. In: Pamiętnik Literacki. 4/2006. Warszawa: Instytut Badań Literackich Polskiej Akademii Nauk - Wydawnictwo, 117142.

Schatte, Cz. 2006. Zu Interphraseologismen in der Mehrsprachigkeitsdidaktik. A. Kątny (ed.) Studia Germanica Gedanensia 14, Gdańsk: Uniwersytet Gdański, 143-156.

Weinrich, H. 1976. Sprache in Texten. Stuttgart: Klett. 